\section{Uncertarnties in the Diagnosis of Position of} Cerebral Tumour.

Mistakes in localisation not infrequently occur, and in this connection may be mentioned those in which no tumour was found at the operation, but after death the tumour was found at the point of operation, sometimes even in the resulting hernia cerebri. This failure may have been due to want of deep exploration of the cerebral substance, but in other cases punctures and incisions were made into the brain but without disclosing the tumour which was present at the spot, Such cases are reported by Dinkler, ${ }^{7}$ Köster, ${ }^{\ngtr}$ and Schultze. ${ }^{9}$ The reasons for this failure should be given by the surgeon. I can only suggest that at present few individual operators are so sufficiently familiar with the aspect and feel of the healthy brain substance as to be always able to differentiate it for tumour growth, especially if this is a soft diffuse glioma. Sometimes a tumour is present on the side expected but not at the spot diagnosed. In the case of a woman at the Manchester Workhouse Infirmary, there were marked and recurring right-sided epileptiform seizures spreading over the face, arm, and leg, together with other symptoms pointing to tumour of the facial area of the left motor cortex. I referred the case to my surgical colleague, Mr. Joseph Collier, for operation, and he agreed that it was a suitable case, at any rate, for exploratory trephining. Before arrangements could be made, however, the patient had several more fits in rapid succession and died. Post mortem a tumour was found in the left second temporo-sphenoidal convolution involving the grey and white matter; the motor cortex appeared normal.

In a case recorded by Schiller, ${ }^{10}$ the tumour was supposed to be in the left arm centre. It was not found at the operation, but at the post-mortem examination one small tumour was found at the posterior part of the left posterior parietal lobule, and a larger wedge-shaped tumour on the median aspect of the left hemisphere in the quadrate lobe, and a cyst in the left corona radiata.

In one of Hitzig's ${ }^{11}$ cases, where trephining over the left motor centre was unsuccessful, a tumour was found post mortem on the median surface of the left hemisphere, the oldest part being in the centrum ovale, and had invaded the gyrus fornicatus and the corpus callosum, and pressed on, but not invaded, the central convolutions.

Difficulties often arise in distinguishing between tumours of the cerebellum and those of the frontal lobe, as illustrated by a case of Hitzig's, ${ }^{12}$ in which a diagnosis of tumour in the right cerebellar hemisphere was made, but post mortem a large cystic glioma of the frontal lobe, flattening the second, third, fifth, and sixth nerves, and the right half of the pons was found. As Bruns ${ }^{13}$ and others have pointed out, ataxia and disturbances of equilibrium are found both in frontal and cerebellar tumours, and a distinction is only possible if symptoms due to pressure on neighbouring structures are present.

In other cases tumours in the internal parts of the brain are mistaken for those near the cortex. Thus Bury, ${ }^{14}$ in a case where the symptoms were giddiness, left-sided paralysis, headache, nausea, blindness, and nystagmus diagnosed a tumour to be subcortical near the motor area, and an unsuccessful operation was performed. The patient died two months afterwards, and a tuberculous tumour of the optic thalamus was found. In Gibson's ${ }^{15}$ case, in which during life there were unilateral spasms and contractions, an operation revealed no tumour, but this was found post mortem in the caudate nucleus and anterior part of the internal capsule. Zeller's ${ }^{15}$ case complained of pain over the left parietal bone, double vision, loss of memory, double choked disc, difficulty in walking, and spasms of the right arm. A tumour of the anterior lower part of the motor region was diagnosed, but no tumour was found at the operation. After a slight improvement the patient became worse and died, and post mortem the tumour was found in the left caudate nucleus, and it sent a process laterally and obliquely upwards into the white matter, but the cortex was not involved.

As I have already mentioned, irritation of the dura mater may cause convulsions on the same side of the body and lead to errors of localisation. In a woman at the Manchester Workhouse Infirmary, suffering from primary carcinoma of the gall bladder with numerous secondary growths, unilateral convulsions on the right side set in, but at the post-mortem examination the only cerebral growth was one limited to the dura mater of the right side.

D. Uncertainty in the Nature of the Tumour.

Intracranial aneurysm may be diagnosed as a tumour or vice versâ, and a distinction is often impossible. Apart from an accurate diagnosis of a tuberculous or syphilitic growth a distinction between the other forms of primary cerebral tumour is often impossible during life. As Gowers says, the diagnosis of the exact nature of the tumour is generally "hardly more than a guess," and " a guess that is almost as likely to be wrong as right." Yet it would be of great benefit if one could distinguish between the diffuse gliomata which are hardly suitable for operation, and the sarcomata which generally have a well-marked limit, whether they spring from bone or membranes, or are within the brain substance, and which can be thus more or less easily "shelled out" at an operation.

It will be seen from the above sketch of a very large subject how difficult it may be for a neurologist to give an accurate opinion on the precise locality and nature of a brain tumour when required to do so by a surgeon before he undertakes an operation; and it can be understood that the difficulties and uncertainties of brain surgery are not so much those of surgical technique or of untoward after-affects, but occur at the outset and with our present knowledge may be well-nigh insurmountable.

REFERENCES.
' Starr, Med. Rec., February ist, i896. 2 Knapp, EPITOMF, BRITISH MEdical Journal, July r6th, r898. 3 Bergmann, Neurol. Centralbl., 1897, p. 920 4 Mills, Transact. Congress Americ. Phys. and Surg., 1888. 5'Pearce Gould, BRITISH MFDICAL JOURNAL. January 22nd, r898. ${ }^{6}$ Phillips, BRITISH MEDICAL JouRNAL, January 22nd, 1898. 7 Dinkle, Neurol. Centralbl., 1897 , p. 6ri. ๖ Köster, Neurol. Centralbl., 1897, p. 1057. 9 Schultz, Deut. Zeits. Nervenheilk., 1896. ${ }^{10}$ Schiller, Neurol. Centralbl., 1897, p. ro56. 11 Hitzig,
Theraps. Wochens., 1896, Nos. 19, 20. 12 Ibid. 13 Bruns, Neurol. Centralbl., Theraps. Wochens., 1896 , Nos. 19, 20.12 Ibid. 13 Bruns, Neurol. Centralbl., ${ }_{15}^{1897}$ Gibson, 922 . 14 Bury, BRITISh Med. Journ., February 7 th, r896. 16 Zeller, Neurol. Centralbl., 1896, p. 288 .

\section{ON THE REMOVAL OF A FRAGMENT OF. STEEL FROM THE RETINA WITH THE ELECTRO-MAGNET,} With Notes of Two Cases.

By SIMEON SNELL, F.R.C.S.EDIN.,

Ophthalmic Surgeon to the Royal Infirmary ; Professor of Ophthalmology, University College, Sheffield.

Since the year $1882,{ }^{1}$ when I described the electro-magnet devised by me for the extraction of splinters of steel or iron from the interior of the eye ${ }^{2}$ many instances have been recorded by $\mathrm{me}^{3}$ and others illustrating the use of the electromagnet in ophthalmic surgery. Its value, and the class of instances in which it is of service, have become generally recognised. The record of isolated cases is hardly, therefore, of interest or value unless there are some special features pertaining to any particular instance. That there are, such, however, in the case related here will at once be recognised. To remove a foreign body from the background of the eye with the retention of perfect sight $\left(\frac{6}{5}\right)$ is a satisfactory event in ophthalmic surgery.

CASE I. Removal of Fragment of Steel from the Retina: Result, Vision $\frac{6}{6}$.
History.-On November 5 th, 1898 , Mi. H. B. M., aged 22 , consulted me on History.- On November 5 th, $1898, \mathrm{Mr}$. H. B. M., aged 22 , consulted me on
account of an injury to his right eye. On October 24 th he was driving a wedge with a hammer, and a splinter flew off and struck the right eye. we cannot say whether the fragment was from the wedge or from the hamHe cannot say whether the fragment the eye at the time and scught medical aid and later he was referred to me.

and later he was referred to ne. Nature of Iniury.-Examination at this visit to me on November 5 th dis closed a small linear wound at the periphery of the cornea, at its outer edge, corresponding to about the centre of the palpebral opening. Beneath this corneal scar a sening in the iris at the per phery, which at once suggested the probability of the foreign body having penetrated and being located in the glcbe. Vision $=6$. The media were clear, but in the fundus towards the periphery on the outer side, just above the median line, a mass of whitish effusion was noticed, in size equal to about 2 diameters of the optic disc. There was only at one point anything resembling the glistening noticeable when a metallic body is embedded in the retina, and this was very trifling. The situation of the effusion corresponded exactly to the line a foreign body passing along the track indicated by the corneal scar and the wound in the iris would take, and no doubt was letina and coated over with this whitisl effusion. 
Operation.-The gravity of the case was pointed out to the patient and to his parents. Consent was obtained to an attempt to remove the foreign body with the electro-magnet, and the operation was performed on November 9 th. The site selected for incising the sclerotic and introducing the electro-magnet was between the external and inferior recti ; ining the electro-magnet was between the external and inferior recti; indeed, a little external to the latter appeared the best situation from which to at once advance the point of the electro-magnet to the location of the
foreign body. Cocaine was therefore instilled, and the conjunctiva separated from a point in front of the intended puncture back towards the rated from a point in front of the intended puncture back towards the
equator. After bleeding was stopped the sclerotic was incised, and the point of the electro-magnet was introduced and advanced to the situation of the foreign body. A second introduction was necessary, and then the piece of steel was extracted; it weighed o. $16 \mathrm{gr}$. One surface was coated white, and this demonstrated, if further testimony were necessary, the fact that it had been situated as diagnosed, one surface lying in the retina
sutures.

After-History.-The recovery was uninterrupted. It is noted on December 16th that there is still a whitish mark where the foreign body was situated, and pigment is becoming deposited in the neighbourhood. Vision $=6$, and the slight alteration in the field of vision, as shown by the accompanying perimeter chart, illustrates how complete has been the restoration of vision.

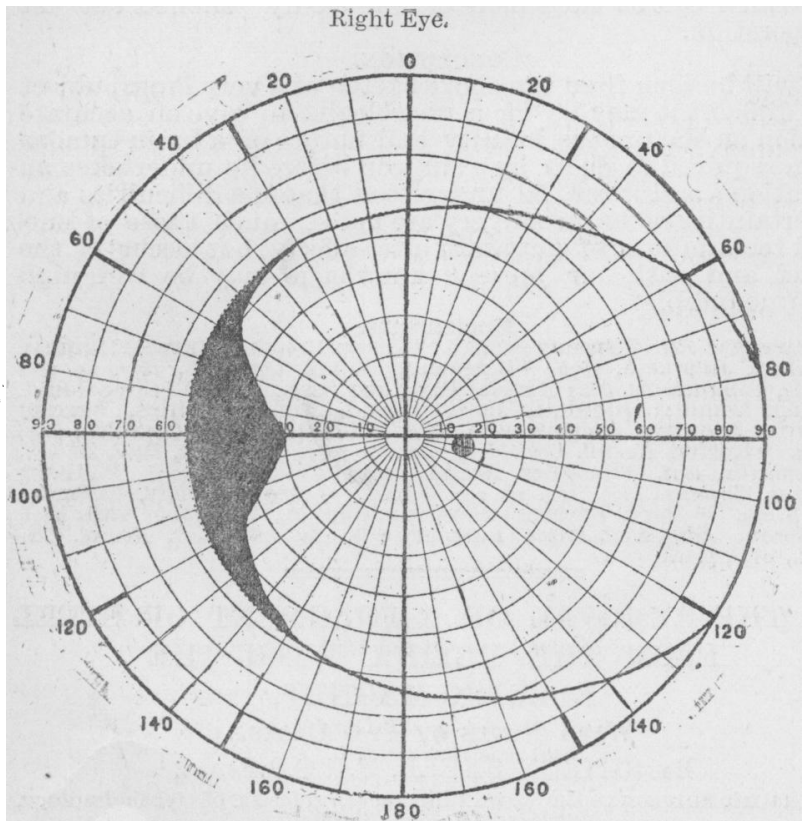

This was the second instance during the year 1898 in which a splinten of steel, diagnosed as being embedded in the retina by the ophthalmoscope, has been successfully removed by me with the electro-magnet. The previous case is recorded in vol. xviii (1898) of the Transactions of the Ophthalmological Society, and reference is also there made to an earlier case of removal of a piece of metal from the retina by me, and to instances recorded by others. The other instance under my care last year very closely resembles that related in the present article.

CASE II. Removal of Fragment of Steel from Retina: Lenticular Opacity : Vision ${ }^{6}$.

History of Injury.-The right eye was injured; the splinter of steel had entered at the sclero-corneal junction at the inner side (not outer), and there was a hole in the iris through which it had passed, and the track the foreign body had taken was made out by the opacity it had caused at the periphery of the lens. Examination with the ophthalmoscope disclosed the fragment of metal fixed in the retina in the lower and outer quadrant. The glistening of the steel was distinct, but it was surrouis wed and partially covered by whitish exudation. The vitreon

Operation and Result.-The sclerotic was incised between the internal Operation and Result. - The sclerotic was incised between the internal and inferior recti, and the electro-magnet was advanced to the situation Again the electro-magnet was passed into the eye and a click was iminediately heard, but with a like result. A careful search was made round the wound to ascertain if the splinter had been peeled off when the electrowound to ascertain withdrawn but it could not be found. A third time the electro-magnet was advanced to the situation of the foreign body : a click was again heard, and this time on withdrawing the instrument the piece of metal was found adhering to it The exact situation of the fragment in the retina had been aseertained, but the splinter was clearly too firmly fixed, either in the retina or glued by adhesions of lymph, to be detached fixed, either in the retina or glued by adhesions of ymph, to be detached magnet. The opacity in lens remained unaltered and vision $=6.6$.

It has for some years been recognised that foreign bodies may be tolerated for lengthened periods in the fundus oculi with retention of vision. F recorded such an instance in 1878 in the Royal London Ophthalmic Hospital Reports ${ }^{4}$ and have reported several instances since," and others, including Knapp, have done the same. Such a case as I have recorded in this paper, together with the other similar instance I have mentioned, very properly raises the question whether foreign bodies so situated should be left alone or surgical interference adopted. My feeling with such evidence before me is. naturally more in favour of interference than it was formerly. But it must be recognised that the fixation or embedding of a foreign body is always a hindrance to its removal by the electro-magnet. In each instance the possibility of success must be carefully weighed, and it is important that the situation of the fragment be exactly located, so that the point of the electro-magnet be advanced immediately to it, and thus the prospects of its successful removal be materially increased.

1 BRITISH MEDICAL JOURNAL, I882, ii. p. 1297. 2 The Electro-magnet and its Employment in Ophthalmic Surgery, Churchill. ${ }^{3}$ BRITISH MEDICAL JoURNAL, 1883, ii, p. 957 ; 1885 , i, p. 884 ; r89o, ii, p. ro55. 4 Royal London Ophthalmic
Hospital Reports, vol. ix, p. 370 . 5 T/ans. Ophthal. Soc., 1886, p. 407; 1893, p. 162 .

\section{A CASE OF PROFUSE SPONTANEOUS HAMOR- RHAGE INTO THE URINARY BLADDER.}

By W. MCADAM ECCLES, M.S.LoND., F.R.C.S.EnG., Assistant Surgeon to the West London Hospital and the City of London

Truss Society, and Demonstrator of Operative Surgery at
St. Bartholomew's Hospital, etc.

VESICAL bleeding may arise from a variety of causes, and blood may reach the bladder from the surrounding structures, but in the majority of instances the amount that is found at any one time in the cavity of the viscus is but slight. Profuse hæmorrhage, leading to a collection of blood so as to distend the bladder, is not a very frequent occurrence, and when it does take place is not altogether simple in its treatment. Such loss of blood into the organ is usually the result of the presence of a papilloma of the vesical wall, but the bleeding may be the outcome of operative interference, or it may be spontaneous.

Malignant disease sometimes causes much hæmorrhage, so likewise a congested prostate. Operations, such as lithotrity and suprapubic cystotomy, are occasionally followed by considerable bleeding. Possibly also hæmophilia must be included as a cause, but it is not usual to get great loss of blood in cases of rupture of the viscus.

The record of the instance of profuse spontaneous hæmorrhage into the bladder which is here given is chiefly of interest from the point of view of treatment.

History.-A man, aged 45, was admitted to the West London Hospital on August 2oth, 1898 , and gave the history of his condition briefly thus: He was a ship-yard painter and had been engaged in painting in a confined place for twelve hours nearly continuously. At the explation of this time he found that he was unable to pass his urine, although up to this period he had never experienced any difficulty whatever in micturition, and there had not been, as far as he knew, anything abnormal with blood water,

with it. Soon after the inability to relieve his bladder came on he began to have a severe pain in the hypogastric region. He returned home, and still being in distress he was seen by his medical man, who passed a catheterwith ease, but failed to draw off more than a few

tient was therefore at once sent into the hospital. Condition on Admission. - When seen there he was evidently in great agony, somewhat collapsed, and distinctly anæmic. On examining the abdomen I found an oval swelling reaching upwards to the level of the umbilicus. This was easily palpated and could be even seen, the patient being of spare habit. The tumour was dull to percussion, but had a rather more firm feel than is usual with a bladder distended with urine. No fluctuation was obtained. The pulse was weak and rapid. A full-sized catheter was passed without difficulty, being introduced its whole length, but only evacuating a small quantity of blood. The finger inserted into the rectum did not reveal that the instrument had transgressed the urethral canal, and there was no evidence of free fiuid in the peritoneal cavity. It was concluded that the case was one of profuse hrmorrhage

into the bladder, and I therefore decided to explore the interior of the
viscus from the perineum.
First Operation -Acordingly, a medium perineal section was made and First Operation. $-A$ ccordingly, a medium perineal section was made and
a catheter introduced through this opening into the bladder. This, when a catheter introduced through this opening into the bladder. This, when it had passed for nearly its full length, drew off about a pint of highly blood-stained urine, and at the same time the hypogastric swelling considerably diminished, but did not entirely disappear. The blood clot
which was in the bladder was as far as possible removed by irrigation,
yet it was elear that all had not been evacuated. The introduction of hot water checked the hæmorrhage almost completely, and a catheter 